\title{
Functions Preserving Orthogonality of Hermitian Matrices
}

\author{
Zongyang Jiang ${ }^{1} \&$ Yang Zhang $^{1}$ \\ ${ }^{1}$ School of Mathematics, Northeast Forestry University, Harbin, China \\ Correspondence: Yang Zhang, School of Mathematics, Northeast Forestry University, Harbin, 150040, China
}

Received: June 1, 2021 Accepted: July 20, 2021 Online Published: July 26, 2021

doi:10.5539/jmr.v13n4p77 URL: https://doi.org/10.5539/jmr.v13n4p77

\begin{abstract}
Inspired by the results that functions preserve orthogonality of full matrices, upper triangular matrices, and symmetric matrices. We finish the work by finding special orthogonal matrices which satisfy the conditions of preserving orthogonality functions. We give a characterization of functions preserving orthogonality of Hermitian matrices.
\end{abstract}

Keywords: functions preserve orthogonality, Hermitian matrix

\section{Introduction}

For an arbitrary field $F, M_{n}(F)$ denotes all $n \times n$ matrices over $F, S_{n}(F)$ denotes all $n \times n$ symmetric matrices over $F$. Let $\mathbb{C}$ and $\mathbb{R}$ be the field of complex numbers and the field of real numbers respectively, meanwhile, $\mathbb{R}^{*}$ be the field of real numbers without 0 . $H_{n}(\mathbb{C})$ denotes all $n \times n$ Hermitian matrices over $\mathbb{C}$. Let $f: \mathbb{C} \rightarrow \mathbb{C}$ be the function such that $A^{f}=\left(f\left(a_{i j}\right)\right)$ when $A=\left(a_{i j}\right)$.

The real motivation of this paper derived from linear preserver problems which have become the major topic in the research of matrix theories. Kalinowski in 2002 proved the theorem (Kalinowski, 2002), i.e. if the function $f: \mathbb{R} \rightarrow \mathbb{R}$ which is odd and strictly increasing satisfies

$$
\operatorname{rank}\left(A^{f}\right)=\operatorname{rank}(A), \forall A \in M_{n}(\mathbb{R})
$$

then there exists a positive constant $c \in \mathbb{R}$ such that

$$
f(x)=c x, \forall x \in \mathbb{R} .
$$

Then in 2003 Kalinowski gave a complete characterization of functions preserving ranks of all matrices, without additional assumption on $f$ (i.e. continuity or others) that is the function $f: \mathbb{R} \rightarrow \mathbb{R}$ preserves rank of matrices if and only if $f(x)=c \cdot x$, where $c \neq 0$ is a constant (Kalinowski, 2003). Hao and Zhang in 2005 generalized the results of Kalinowski and gave the theorem (Hao \& Zhang, 2005),i.e. for any fixed integer $k$ which satisfies $2 \leq k<n$, the function $f: F \rightarrow F$ satisfies

$$
\operatorname{rank}(A)=k \Rightarrow \operatorname{rank}\left(A^{f}\right)=k, A \in M_{n}(F)
$$

if and only if there exist a non-zero scalar $c$ and an injective field endomorphism $\delta$ of $F$ such that

$$
f=c \delta .
$$

Fan in 2015 used the results of Hao and Zhang, characterized the function preserving orthogonality on the spaces of full matrices, upper triangular matrices, and symmetric matrices,respectively (Fan, 2015), if and only if the function satisfies

$$
f=c \delta
$$

where $c \in F, \delta$ is an injective field endomorphism.

The purpose of this paper is to find new Hermitian matrices that satisfied the conditions of function preserving orthogonality, which can further enrich function preservation problems. The idea of Hermitian matrices is inspired by the thesis (Tang \& Cao, 2005).

\section{Preliminaries}

The following definitions and lemma can be resulted from the paper (Fan, 2015).

Definition 1 The $n \times n$ matrices $A$ and $B$ is orthogonal if $A, B \in M_{n}(F), A B=B A=0$.

Definition 2 The function $f: \mathbb{C} \rightarrow \mathbb{C}$ preserves orthogonality on the spaces of Hermitian matrices over field if $f$ satisfies

$$
A B=B A=0 \Longrightarrow A^{f} B^{f}=B^{f} A^{f}=0, \forall A, B \in H_{n}(\mathbb{C}) ;
$$


Definition 3 The function $f: \mathbb{C} \rightarrow \mathbb{C}$ is the field endomorphism if $f$ satisfies

$$
f(\alpha+\beta)=f(\alpha)+f(\beta), f(\alpha \beta)=f(\alpha) f(\beta), \forall \alpha, \beta \in \mathbb{C} .
$$

Lemma 1 The function $f$ preserves orthogonality on the spaces of $S_{n}(F)$ if and only if $f=c \delta$, where $c \in F, \delta$ is the injective field endomorphism.

\section{Main Result}

Theorem 1 The function $f$ preserves orthogonality of $H_{n}(\mathbb{C})(n \geq 3)$ if and only if $f=c \delta$, where $c \in \mathbb{C}, \delta$ is the injective field endomorphism.

Proof. Since the sufficiency part is obvious, we consider only the necessary part. As $f \equiv 0$ is satisfied, the discussion is divided into the following three cases assuming that $f \neq 0$.

Case $1 f\left(b_{1} i+b_{2} i\right)=f\left(b_{1} i\right)+f\left(b_{2} i\right), f(1) f\left(b_{1} i \cdot b_{2} i\right)=f\left(b_{1} i\right) f\left(b_{2} i\right)$, for all $b_{1}, b_{2} \in \mathbb{R}$

For any $x \in \mathbb{R}$, let $A_{1}=\left[\begin{array}{ccc}x & x i & 0 \\ -x i & x & 0 \\ 0 & 0 & 0\end{array}\right] \oplus 0, B_{1}=\left[\begin{array}{ccc}x & -x i & 0 \\ x i & x & 0 \\ 0 & 0 & 0\end{array}\right] \oplus 0, A_{1}, B_{1} \in H_{n}(\mathbb{C})$, thus $A_{1} B_{1}=B_{1} A_{1}=0$. By the definition of $f$ and calculation, we obtain

$$
\begin{gathered}
f(0)=0 \\
f^{2}(0)+f^{2}(x)+f^{2}(x i)=0
\end{gathered}
$$

Through analyzing it follows from $f(x)=0$ and (2) that $f(x i)=0$. It implies that $f \equiv 0$ which conflicts with the assumption $f \neq 0$. From the above, it follows that $f(x) \neq 0$ and thus $f(x i) \neq 0$, when $x \neq 0$.

For any $x \in \mathbb{R}$, let $A_{2}=\left[\begin{array}{ccc}1 & 1 & i \\ 1 & 1 & i \\ -i & -i & 1\end{array}\right] \oplus 0, B_{2}=\left[\begin{array}{ccc}-x & 0 & x i \\ 0 & x & -x i \\ -x i & x i & 0\end{array}\right] \oplus 0, A_{2}, B_{2} \in H_{n}(\mathbb{C})$, thus $A_{2} B_{2}=B_{2} A_{2}=0$. Then we obtain

$$
f(0) f(i)+f(1)[f(x i)+f(-x i)]=0
$$

From the above $f(0)=0, f(1) \neq 0$, we have

$$
f(-x i)=-f(x i)
$$

Let $A_{3}=\left[\begin{array}{ccc}1 & 1 & -b_{1} i \\ 1 & 1 & -b_{1} i \\ b_{1} i & b_{1} i & b_{1}^{2}\end{array}\right] \oplus 0, B_{3}=\left[\begin{array}{ccc}b_{1}^{2}+2 b_{1} b_{2} & -b_{1} b_{2} & \left(b_{1}+b_{2}\right) i \\ -b_{1} b_{2} & 0 & -b_{2} i \\ -\left(b_{1}+b_{2}\right) i & b_{2} i & 1\end{array}\right] \oplus 0, A_{3}, B_{3} \in H_{n}(\mathbb{C})$, thus $A_{3} B_{3}=B_{3} A_{3}=0$

Then we obtain

$$
f(1)\left[f\left(\left(b_{1}+b_{2}\right) i\right)+f\left(-b_{1} i\right)+f\left(-b_{2} i\right)\right]=0
$$

As $f(1) \neq 0$ and (4), we have

$$
f\left(b_{1} i+b_{2} i\right)=f\left(b_{1} i\right)+f\left(b_{2} i\right)
$$

Let $A_{4}=\left[\begin{array}{ccc}b_{1}^{2} & -b_{1} i & -b_{1} i \\ b_{1} i & 1 & 1 \\ b_{1} i & 1 & 1\end{array}\right] \oplus 0, B_{4}=\left[\begin{array}{ccc}-1 & -\left(b_{1}+b_{2}\right) i & b_{2} i \\ \left(b_{1}+b_{2}\right) i & -b_{1}\left(b_{1}+b_{2}\right) & 0 \\ -b_{2} i & 0 & b_{1} b_{2}\end{array}\right] \oplus 0, A_{4}, B_{4} \in H_{n}(\mathbb{C})$, thus $A_{4} B_{4}=B_{4} A_{4}=0$.

By the direct calculation, we obtain

$$
f(1) f(0)+f(1) f\left(b_{1} b_{2}\right)+f\left(b_{1} i\right) f\left(b_{2} i\right)=0
$$

With (4) and $f(0)=0$, we have

$$
f(1) f\left(b_{1} i \cdot b_{2} i\right)=f\left(b_{1} i\right) f\left(b_{2} i\right)
$$

Let $\delta=f^{-1}(1) f$, we use (6) such that

$$
\delta\left(b_{1} i+b_{2} i\right)=f^{-1}(1) f\left(b_{1} i+b_{2} i\right)=f^{-1}(1) f\left(b_{1} i\right)+f^{-1}(1) f\left(b_{2} i\right)=\delta\left(b_{1} i\right)+\delta\left(b_{2} i\right)
$$

Applying (8), we can get

$$
\delta\left(b_{1} i \cdot b_{2} i\right)=f^{-1}(1) f\left(b_{1} i \cdot b_{2} i\right)=f^{-1}(1) f^{-1}(1) f\left(b_{1} i\right) f\left(b_{2} i\right)=\delta\left(b_{1} i\right) \delta\left(b_{2} i\right)
$$


With (8) we have $f^{2}(1)=f(1) f\left(b i \cdot(b i)^{-1}\right)=f(b i) f\left((b i)^{-1}\right) \neq 0, \forall b \in \mathbb{R}^{*}$, hence we obtain

$$
\forall b \in \mathbb{R}^{*}, f(b i) \neq 0
$$

If $\delta\left(b_{1} i\right)=\delta\left(b_{2} i\right)$, we conclude that $b_{1} i=b_{2} i$ by (4), (6), (9) and (11).

Case $2 f\left(a_{1}+a_{2}\right)=f\left(a_{1}\right)+f\left(a_{2}\right), f(1) f\left(a_{1} a_{2}\right)=f\left(a_{1}\right) f\left(a_{2}\right)$, for all $a_{1}, a_{2} \in \mathbb{R}$

The following conclusions are obtained immediately from the proof of Lemma 1

$$
\begin{gathered}
f\left(-a_{1}\right)=-f\left(a_{1}\right) \\
f\left(a_{1}+a_{2}\right)=f\left(a_{1}\right)+f\left(a_{2}\right)
\end{gathered}
$$

For any $a_{1}, a_{2}, c \in \mathbb{R}$, let $A_{5}=\left[\begin{array}{ccc}1 & c i & a_{2} \\ -c i & c^{2} & -a_{2} c i \\ a_{2} & a_{2} c i & a_{2}^{2}\end{array}\right] \oplus 0, B_{5}=\left[\begin{array}{ccc}c^{2}-a_{1} a_{2}^{2} & -c i & a_{1} a_{2} \\ c i & 1 & 0 \\ a_{1} a_{2} & 0 & -a_{1}\end{array}\right] \oplus 0, A_{5}, B_{5} \in H_{n}(\mathbb{C})$, thus $A_{5} B_{5}=B_{5} A_{5}=0$. Then we have

$$
f(1) f\left(a_{1} a_{2}\right)+f(c i) f(0)+f\left(-a_{1}\right) f\left(a_{2}\right)=0
$$

Applying (12) and $f(0)=0$, we can get

$$
f(1) f\left(a_{1} a_{2}\right)=f\left(a_{1}\right) f\left(a_{2}\right)
$$

Let $\delta=f^{-1}(1) f$, we use (13) such that

$$
\delta\left(a_{1}+a_{2}\right)=f^{-1}(1) f\left(a_{1}+a_{2}\right)=f^{-1}(1) f\left(a_{1}\right)+f^{-1}(1) f\left(a_{2}\right)=\delta\left(a_{1}\right)+\delta\left(a_{2}\right)
$$

Applying (15), we obtain

$$
\delta\left(a_{1} a_{2}\right)=f^{-1}(1) f\left(a_{1} a_{2}\right)=f^{-1}(1) f^{-1}(1) f\left(a_{1}\right) f\left(a_{2}\right)=\delta\left(a_{1}\right) \delta\left(a_{2}\right)
$$

With (15), we have $f^{2}(1)=f(1) f\left(a \cdot a^{-1}\right)=f(a) f\left(a^{-1}\right) \neq 0, \forall a \in \mathbb{R}^{*}$, hence we obtain

$$
\forall a \in \mathbb{R}^{*}, f(a) \neq 0
$$

If $\delta\left(a_{1}\right)=\delta\left(a_{2}\right)$, we conclude that $a_{1}=a_{2}$ by (12), (13), (16) and (18).

Case $3 f\left(a_{1}+b_{2} i\right)=f\left(a_{1}\right)+f\left(b_{2} i\right), f(1) f\left(a_{1} \cdot b_{2} i\right)=f\left(a_{1}\right) f\left(b_{2} i\right)$, for all $a_{1}, b_{2} \in \mathbb{R}$

Let $A_{6}=\left[\begin{array}{ccc}1 & -b_{2} i & 1 \\ b_{2} i & b_{2}^{2} & b_{2} i \\ 1 & -b_{2} i & 1\end{array}\right] \oplus 0, B_{6}=\left[\begin{array}{ccc}-a_{1} & -1 & a_{1}+b_{2} i \\ -1 & 0 & 1 \\ a_{1}-b_{2} i & 1 & -a_{1}\end{array}\right] \oplus 0, A_{6}, B_{6} \in H_{n}(\mathbb{C})$, thus $A_{6} B_{6}=B_{6} A_{6}=0$. By the direct calculation, we obtain

$$
f(1)\left[f\left(a_{1}+b_{2} i\right)+f\left(-a_{1}\right)+f\left(-b_{2} i\right)\right]=0
$$

Applying (4), (12) and $f(1) \neq 0$, we have

$$
f\left(a_{1}+b_{2} i\right)=f\left(a_{1}\right)+f\left(b_{2} i\right)
$$

Let $A_{7}=\left[\begin{array}{ccc}1 & 1 & b_{2} i \\ 1 & 1 & b_{2} i \\ -b_{2} i & -b_{2} i & b_{2}^{2}\end{array}\right] \oplus 0, B_{7}=\left[\begin{array}{ccc}0 & -a_{1} b_{2}^{2} & a_{1} b_{2} i \\ -a_{1} b_{2}^{2} & a_{1} b_{2}^{2} & 0 \\ -a_{1} b_{2} i & 0 & -a_{1}\end{array}\right] \oplus 0, A_{7}, B_{7} \in H_{n}(\mathbb{C})$, thus $A_{7} B_{7}=B_{7} A_{7}=0$. We obtain

$$
f(1) f\left(a_{1} \cdot b_{2} i\right)+f\left(-a_{1}\right) f\left(b_{2} i\right)=0
$$

Applying (12), we have

$$
f(1) f\left(a_{1} \cdot b_{2} i\right)=f\left(a_{1}\right) f\left(b_{2} i\right)
$$

Let $\delta=f^{-1}(1) f$, we use (20) such that

$$
\delta\left(a_{1}+b_{2} i\right)=f^{-1}(1) f\left(a_{1}+b_{2} i\right)=f^{-1}(1) f\left(a_{1}\right)+f^{-1}(1) f\left(b_{2} i\right)=\delta\left(a_{1}\right)+\delta\left(b_{2} i\right)
$$


Applying (22), we have

$$
\delta\left(a_{1} \cdot b_{2} i\right)=f^{-1}(1) f\left(a_{1} \cdot b_{2} i\right)=f^{-1}(1) f^{-1}(1) f\left(a_{1}\right) f\left(b_{2} i\right)=\delta\left(a_{1}\right) \delta\left(b_{2} i\right)
$$

Next let us prove $\delta$ is injective.

If $\delta\left(a_{1}+b_{1} i\right)=\delta\left(a_{2}+b_{2} i\right)$, we can conclude that $a_{1}+b_{1} i=a_{2}+b_{2} i$ which follows from (23) and the discussion of case 1 and case 2 .

In conclusion, the necessity is proved, that is $f=c \delta$, where $c=f(1), \delta$ is the injective field endomorphism.

\section{Conclusion}

The problem of functions preserving orthogonality of Hermitian matrices has proved. But there are other matrices like skew symmetric matrices and skew Hermitian matrices have no researched which we can attempt to do for further study.

\section{References}

Kalinowski, J. (2002). On rank equivalence and rank preserving operators. Novi Sad J. Math., 32, 133-139.

Kalinowski, J. (2003). On functions preserving rank of matrices. Math Notes, 4(1), 35-37. https://doi.org/10.18514/MMN.2003.6

Hao, L., \& Zhang, X. (2005). Functions preserving rank- $k$ matrices of order $n$ over fields. Miskolc Mathematical Notes, 2(2), 197-200. https://doi.org/10.18514/MMN.2005.100

Cao, C., \& Xian, Z. (1996). Additive operators preserving idempotent matrices over fields and applications. Linear Algebra and Its Applications, 248(248), 327-338. https://doi.org/10.1016/0024-3795(96)89195-6

Hong-mei, Y., Xiao-cui, S., \& Guang-hui, W. (2011). A Note on Functions Preserving some Properties of Matrices. Proceeding of the Sixth International Conference of Matrices and Operators, 2, 87-90.

Chi-Kwong, P., \& Stephen. (2001). Linear preserver problems. American Mathematical Monthly, 108(7), 591-591. https://doi.org/10.1080/00029890.2001.11919790

Li-jie, G., \& Chong-guang, C. (2011). Functions Preserving Square-zero Matrices of Order n Over Fields. Proceeding of the Sixth International Conference of Matrices and Operators, 1, 226-228.

Fan, Y., Xiaofeng, M. A., Tan, L. (2015). Function preserving orthogonal matrix over the field of characteristic 0. Journal of Natural Science of Heilongjiang University, 32(1), 54-57. https://doi.org/10.13482/j.issn1001-7011.2014.11.276

Tang, X. M., \& Cao, C. G. (2005). Linear maps preserving pairs of Hermitian matrices on which the rank is additive and applications. Journal of Applied Mathematics and Computing, 19(1-2), 253-260. https://doi.org/10.1007/BF02935803

Liu, Y., \& Tian, Y. (2010). Extremal ranks of submatrices in an Hermitian solution to the matrix equation AXA*=B with applications. Journal of Applied Mathematics and Computing, 32(2), 289-301. https://doi.org/10.1007/s12190-0090251-8

Liu, Y., Tian, Y., \& Takane, Y. (2009). Ranks of Hermitian and skew-Hermitian solutions to the matrix equation AXA*=B. Linear Algebra and Its Applications, 431(12), 2359-2372. https://doi.org/10.1016/j.laa.2009.03.011

Li, N., Jiang, J., \& Wang, W. F. (2011). Hermitian solutions to a quaternion matrix equation. Applied Mechanics and Materials, 50-51, 391-395. https://doi.org/10.4028/www.scientific.net/AMM.50-51.391

Rehman, A., \& Akram, M. (2017). Optimization of a nonlinear Hermitian matrix expression with application. Filomat, 31(9), 2805-2819. https://doi.org/10.2298/FIL1709805R

Guterman, A. E., \& Spiridonov, I. A. (2020). Permanent polya problem for additive surjective maps. Linear Algebra and its Applications, 599. https://doi.org/10.1016/j.laa.2020.04.001

\section{Copyrights}

Copyright for this article is retained by the author(s), with first publication rights granted to the journal.

This is an open-access article distributed under the terms and conditions of the Creative Commons Attribution license (http://creativecommons.org/licenses/by/4.0/). 